\title{
40 Jahre Hamburger Friedensforschung. Das IFSH 1971-2011
}

\author{
Detlef Bald*
}

$\mathrm{E}$ in beachtlicher, ein beachtenswerter Weg wurde vom Institut für Friedensforschung und Sicherheitspolitik an der Universität Hamburg beschritten, 40 Jahre Institut, eine lange Geschichte - wer hätte bei der Gründung am 11. Juni 1971 an diese Zukunft des IFSH gedacht? ${ }^{1}$ Vor 40 Jahren, als das Institut im Herbst 1971 „seine Tore geöffnet“ hatte, begleiteten die ersten drei Wissenschaftler des Hauses den „Start vom Punkt Null“" enthusiastisch mit größter Hoffnung - wie in einer freundschaftlich verbundenen, großen Familie. ${ }^{2}$ Das war am Anfang, als das Institut, stromabwärts und abgeschieden in den malerischen Wäldern der hügeligen Uferhänge der Elbe hinter Blankenese gelegen, am Falkenstein aufgebaut wurde.

Hamburg wurde seinem Ruf gerecht, eine dem Handel über die Meere zugewandte, weltoffene Stadt zu sein, als es dieses Projekt eines „Spezialinstituts“ anging, „sich um die Lösung weitgespannter Fragenkomplexe der Gestaltung der Zukunft“ zu bemühen; das war in der Hochzeit des Kalten Krieges und der nuklearen Bedrohung mehr als notwendig. Nur Frieden konnte Zukunft gewähren, Handel benötigt Frieden. „Zukunftsforschung“: die „vielen Einzelfragen der Sicherheitspolitik wissenschaftlich erarbeiten zu können“ - so stellte sich die Hansestadt mit klarer Aussage an die Spitze der Wissenschaftspolitik in Deutschland und legte ein Konzept „der freien Grundlagenforschung“von „überregionaler Bedeutung “vor. Sie stellte sich in eine bekannte Tradition: „Die Hansestädte sind ein gutes Pflaster für Friedenspolitik. "3 Lapidar wurde festgestellt, diese Metropole „eignet sich besonders gut als Standort für ein derartiges Spezialinstitut“. ${ }^{4}$ Das klang ein wenig euphorisch und entsprach der Hoffnung zu Beginn der Planungen im Oktober 1969, das IFSH bald zu eröffnen, bevor stadtpolitische Auseinandersetzungen eine doch längere Gründungsphase verlangten.

Das Institut feierte im Herbst 2011 das Jubiläum seines 40-jährigen Bestehens. Die Glückwünsche dazu sind berechtigt: Das Institut hat breite Forschung betrieben und die Friedens- und Konfliktforschung mit reichen Impulsen vertreten und vorangetrieben; es hat in die wissenschaftlichen Nachbardisziplinen hineingewirkt, in Deutschland hat sein Name einen guten Klang und verbürgt anerkannte Reputation. International ist es hoch respektiert - schon Anlass genug Dank zu sagen, dem

* Dr. Detlef Bald, vormals Wissenschaftlicher Direktor für „Militär und Gesellschaft" am Sozialwissenschaftlichen Institut der Bundeswehr, ist Historiker, Politikwissenschaftler und Publizist.

1 Der Text basiert auf einem Vortrag, den Detlef Bald am 14. November 2011 anläßlich des 40jährigen Bestehens des IFSH in Hamburg gehalten hat.

2 BDZ (Baudissin Dokumentationszentrum, Hamburg) 803001 Gräfin Dagmar Baudissin an B., 26. Nov. 1971.

3 Gernot Erler: Friedenspolitisches handeln vor dem Hintergrund regionaler und globaler Herausforderungen, in: Hans Kloft (Hg.): Friedenspolitik und Friedensforschung. Die Friedensnobelpreisträger aus Deutschland, Berlin 2011, S. 79.

4 BDZ 809133 Freie und Hansestadt Hamburg, Schulbehörde an Stiftung Volkswagenwerk, 16. Okt. 1969. ganzen Haus, den Kolleginnen und Kollegen, die es eigentlich gestaltet haben. Sie haben die Leistungen erbracht und den Ruf des IFSH begründet; sicher gab es einige, die es mehr prägten als andere. Doch einzelne Personen und Leistungen in diesem Zusammenhang hervorzuheben hieße auch, andere zurückzusetzen. Sie alle mit ihren Leistungen zu würdigen, müsste an anderer Stelle und systematisch geschehen.

Und doch sehe ich mich verpflichtet zu sagen: Dieses Institut wäre ohne seine ehemaligen Direktoren nicht zu dem geworden, was heute Anlass ist zu erinnern. Die jeweils ganz eigenen, die starken Persönlichkeiten mit Weitblick und Courage, mit Weltläufigkeit und Kompetenz: Wolf Graf von Baudissin, Egon Bahr und Dieter S. Lutz - ihre Namen sind mit diesem Haus und seiner Geschichte aufs innigste verbunden. Ihre Autorität beruhte auf analytischen, realistischen und zugleich visionären Eigenschaften, die faszinierten. Sie haben Identität und Präsenz geschaffen; sie haben Entwicklungen vorangetrieben oder auch nur großzügig zugelassen. Sie haben die Freiheit der Forschung geschützt und haben sich der Öfentlichkeit gestellt und doch oft genug auch vor die Mitarbeiterinnen und Mitarbeiter. Sie haben dem IFSH Form und Format gegeben. Es ist schon ein Glück, dass es sie gab. ${ }^{5}$

\section{Zum politischen Impuls des Bundespräsidenten}

Was bot vor 40 Jahren den Anlass für wissenschaftliche Innovationen, was führte zur Gründung des Instituts? Es war eine Zeit politischer Unruhe, eine historische Wende in der noch jungen Geschichte der Bundesrepublik zeichnete sich ab. Gustav Heinemann, der erste sozialdemokratische Bundespräsident in Bonn, hatte in seiner Antrittsrede vor Bundestag und Bundesrat am 1. Juli 1969 gerade für Verständigung nach Osten und Entspannung in der Außen- und Sicherheitspolitik geworben und ausgeführt: „Ich sehe als erstes die Verpflichtung, dem Frieden zu dienen. Nicht der Krieg ist der Ernstfall, in dem der Mann sich zu bewähren habe, wie meine Generation in der kaiserlichen Zeit auf den Schulbänken lernte, sondern der Frieden ist der Ernstfall, in dem wir uns alle zu bewähren haben. Hinter dem Frieden gibt es keine Existenz mehr. “6

Heinemann ließ aufhorchen mit der Mahnung, „Frieden“ ernst zu nehmen und für die aktuelle Politik grundlegend anzunehmen. Seine berühmte Formel vom „Ernstfall Frieden“ aber „ermutigte“ auch Baudissin und gab den Anstoß, dieses Friedensforschungsinstitut aufzubauen. ${ }^{7}$ Genau diese Inten-

5 Vgl. Das Institut für Friedensforschung und Sicherheitspolitik an der Universität Hamburg, hrsg. aus Anlaß des 25-jährigen Bestehens des IFSH, Hamburg 1997.

6 Gustav W. Heinemann: Reden und Interviews, Bd. I, Bonn (o.J.), S. 9ff.

7 BDZ 809109 Wolf Graf von Baudissin, Abschiedsvorlesung an der Universität der Bundeswehr Hamburg, 18. Juni 1986, S. 30. 
tion verfolgte Heinemann, als er für Deutschland forderte: „Hilfreich wäre es, wenn auch wir der Friedensforschung, das heißt einer wissenschaftlichen Ermittlung nicht nur der militärischen Zusammenhänge zwischen Rüstung, Abrüstung und Friedenssicherung, sondern zwischen allen Faktoren, also z.B. auch den sozialen, den wirtschaftlichen und den psychologischen die gebührende Aufmerksamkeit zuwenden würden."

Dieses Monitum des Bundespräsidenten bewegte die Gemüter, es führte innenpolitisch zu einem Aufruhr der Abgrenzung in der Gesellschaft und emotionalisierte die parteipolitischen Kontroversen, besonders zu einer scharfen Gegnerschaft in der Bildungspolitik. In militärischen und konservativen Kreisen wurde Heinemanns Rede vom „Ernstfall Frieden“ geradezu als ungeheuerliche Provokation empfunden, während er in der seinerzeit hochgradig politisierten Bevölkerung mit der Zustimmung vieler Zeitgenossen rechnen konnte. Angesichts des verbreiteten Bewusstseins, Sicherheit im Atomzeitalter sei nur unter den Bedingungen einer militärischen Eskalation hin zum Atomkrieg zu gewährleisten, musste es die alles überragende Aufgabe der Politik sein, eine solche Katastrophe zu verhindern. Millionen von Menschen fühlten sich durch die Worte Heinemanns in ihrer Sorge um die Sicherheit gegenüber dem Abschreckungsfrieden der nuklearen Bedrohung ernst genommen.

Der Bundespräsident hatte das deutsche Defizit der Friedensforschung zu Recht angesprochen, da sie in anderen Ländern längst universitär etabliert und politisch respektiert war. Die Explosion der ersten Atombombe 1945 bildete die Zäsur. Amerikanische Naturwissenschaftler hatten vor diesen Zerstörungspotenzialen gewarnt und suchten sie einzuhegen. ${ }^{8}$ Noch verhallte der Ruf von Albert Einstein einsam, im Zeitalter der Nuklearwaffen benötige die Wissenschaft ein substanziell neues Denken, eine neue Denkungsart, wenn die Menschheit überleben wolle. Friedensforschung ist daher an eine Friedensethik gebunden, die mit dem Atomzeitalter verknüpft ist. Erstmals in der Geschichte konnten seit Hiroshima militärische Zerstörungspotenziale - eine ungeheuerliche, globale Dimension die Vernichtung der Menschheit bewirken.

Mahnende Worte, große Reden und Proklamationen sowie wichtige Bücher, die warnend auf die nukleare Aufrüstung hinwiesen, hatten die fünfziger Jahre begleitet. Doch die Wissenschaften blieben außen vor. Nicht zufällig signalisiert daher die Rede von Carl Friedrich von Weizsäcker aus dem Jahre 1963 über die „Bedingungen des Friedens im technischen Zeitalter" einen Einschnitt und gilt manchen als Beginn der friedenswissenschaftlichen Arbeiten einzelner Forscher. ${ }^{9} \mathrm{Zu}$ ihnen gehörten einige Namen, die schon in den sechziger Jahren mit Publikationen hervortraten und bedeutsam für die damalige Orientierung in Theorie und Diskussion in den Politik-, Sozial- und Geisteswissenschaften wurden: Horst Afheldt, Ernst-Otto Czempiel, Theodor Ebert, Erhard Forndran, Egbert Jahn, Karl Kaiser, Ekkehart Krippendorff, Georg Picht, Dieter

8 Vgl. Detlef Bald: Hiroshima 6. August 1945. Die nukleare Bedrohung, München 1999, S. 29ff.; Ekkehart Krippendorff (Hg.): Friedensforschung, Köln 1974; Corinna Hauswedell: Friedenswissenschaften im Kalten Krieg. Friedenswissenschaften und friedenswissenschaftliche Initiativen in der Bundesrepublik Deutschland in den achtziger Jahren, Baden-Baden 1997, S. 37ff.

9 Abgedruckt in: Carl Friedrich von Weizsäcker: Der bedrohte Frieden. Politische Aufsätze 1945-1981, München 1983, S. $125 \mathrm{ff}$.
Senghaas, Fritz Vilmar - und natürlich ist ebenso Johan Galtung zu nennen, der auch in den folgenden Jahrzehnten gerade hier am IFSH seinen Beitrag leistete, Strukturen von Gewalt im Ost-West-Konflikt zu begreifen, aber auch hervortrat, um den Blick auf die Macht des Nordens gegenüber dem Süden zu richten. ${ }^{10}$

\section{Graf Baudissin - ein General für den Frieden}

In Hamburg wurde der erste Direktor des Instituts für Friedensforschung und Sicherheitspolitik nicht zufällig berufen. Sicher, er war Generalleutnant auch an führender Stelle in der NATO gewesen - was wohl keine hinlängliche Qualifikation für ein Institut der Sicherheitspolitik, erst recht nicht der Friedensforschung ist, wenn man die übliche sicherheitspolitische Kompetenz von Offizieren im Generalstabsdienst annimmt. Doch Wolf Graf Baudissin war anders. Er war gewiss kein militärischer Handlanger, kein Techniker der Gewalt. Er kann auch nicht angemessen als militärischer Querdenker oder intellektueller Vordenker bezeichnet werden - wie man gerne ungewöhnliche Fähigkeiten umschreibt. Das trifft nicht den Kern, warum es eine glückliche Fügung war, dass er Gründungsdirektor des IFSH wurde. Ihn zeichnete ungewöhnliche Sachkenntnis in rebus militariis wie kaum einen anderen aus. Das gilt zunächst für die sicherheitspolitische Analyse, die er in den frühen sechziger Jahren in Bonn kennenlernte, militärische Profession mit wissenschaftlicher Expertise zu unterlegen und zu prüfen. Er war beeindruckt vom Vorbild der direkten Politikberatung in den USA, wissenschaftliche Kompetenz zu bündeln, um politische Probleme der Sicherheit und Rüstung zu lösen. Dort hatte gerade John F. Kennedy in Washington vorgemacht, die bipolare Konfrontation durch Kooperation wissenschaftlicher Experten (erste Pugwash-Konferenz in Moskau 1960) ${ }^{11}$ abzubauen, um wechselseitiges Vertrauen aufzubauen. Eigene Schlüsse aus diesen Ergebnissen zog ein umtriebiger junger Politiker, der als Hamburger Innensenator das damals beste Buch zur Bündnispolitik verfasst hatte. ${ }^{12}$ Helmut Schmidt suchte in Bonn die sicherheitspolitischen Verkrustungen durch Expertenrunden aus Politik, Militär und Wissenschaft aufzubrechen; Nutzen und Vorteil hatten die "Stiftung Wissenschaft und Politik“ sowie die „Deutsche Gesellschaft für Auswärtige Politik“. Schmidt, einer der Initiatoren, lernte dabei Graf Baudissin kennen, der ebenso, um nur einen weiteren zu nennen, nämlich Carl Friedrich von Weizsäcker in der folgenden Zeit zu den vertraulichen Gesprächskreisen in Hamburg um Marion Gräfin Dönhoff eingeladen wurde. ${ }^{13}$

Mit einer brisanten Veröffentlichung hatte Baudissin als aktiver General seine sicherheitspolitische Kenntnis sowie friedensethische Grundhaltung schon 1962 zu erkennen gegeben, als er

10 Vgl. Ulrike C. Wasmuht: Geschichte der deutschen Friedensforschung. Entwicklung - Selbstverständnis - Politischer Kontext, Münster 1998, S. 117ff.

11 Vgl. Bernd W. Kubbig: Kommunikatoren im Kalten Krieg. Die PugwashKonferenzen, die US-Sowjetische Studiengruppe und der ABM-Vertrag, Frankfurt/M. 1996.

12 Vgl. Helmut Schmidt: Verteidigung oder Vergeltung. Ein deutscher Beitrag zum strategischen Problem der Verteidigung, Stuttgart 1961 und später: Strategie des Gleichgewichts, Stuttgart 1969.

13 Vgl. Detlef Bald: Politik der Verantwortung. Das Beispiel Helmut Schmidt. Der Primat des Politischen über das Militärisch 1965-1975, Berlin 2008, S. 70 ff. 
das atomare Kriegsbild in Konsequenz an sein Ende führte und die alles vernichtende „Totalität“ des Krieges mit den berühmt gewordenen Worten brandmarkte: „... von da an herrscht Friedhofsruhe“.${ }^{14}$ Klar zeigte er auf, Verteidigung mit Atomwaffen bedeutet Selbstvernichtung. Baudissins skeptische Haltung wäre ohne seine verantwortungsethische Fundierung kaum zu begreifen, die ihre Nähe zur alten protestantischen, lutherisch geprägten Lehre spüren lässt, dass ein Land sich verteidigen könne, aber dass es dem Frieden verpflichtet sei. Dies hatte sich bei seiner Mitwirkung an den „Heidelberger Thesen“ von 1959 bereits gezeigt, als die evangelische Kirche den Atomwaffeneinsatz nicht mehr als Ultima Ratio der Verteidigung bezeichnete, sondern nuklear gestützte Verteidigung nur „noch“ als ein Mittel der Friedenssicherung eingrenzte: Politik habe dem Frieden zu dienen und diese Atomwaffen abzurüsten. ${ }^{15}$ Doch die angestrebte neue Denkungsart, die nukleare Verteidigung weiter zu ächten, blieb weiterhin eine friedenspolitische Aufgabe.

\section{Die lange Gründungsphase}

Als Baudissin dann im Oktober 1969 den Lehrauftrag über „moderne Strategie“ an der Universität Hamburg übernahm, bot er eine akademische „Beschäftigung mit strategischen Fragen“ an, die „nichts anderes sein“ sollte, wie er betonte, „als ein bescheidenes Korrelat zur Friedensforschung “. ${ }^{16} \mathrm{Zu}$ diesem Zeitpunkt und drei Monate nach der Rede Heinemanns - am 22. September 1969 - stellte er den Antrag an die Stiftung Volkswagenwerk für den Aufbau des, so lautete seine Namensgebung, „Instituts für Sicherheitspolitik an der Universität Hamburg“. Er warf den ersten Stein ins Wasser, der weitreichende Wellen erzeugte. Hamburg schloss sich dieser Initiative am 16. Oktober mit einem vollständigen Konzeptantrag an; nach intensiven Erörterungen zwischen der Behörde und Baudissin wurden Ausrichtung und Struktur des Instituts umrissen. Erstmals wurde der bis heute gültige Name des Instituts in einem Schreiben der Stadt Hamburg an den Rektor der Universität am 9. Dezember 1969 benannt: „Institut für Friedensforschung und Sicherheitspolitik an der Universität Hamburg“" ${ }^{17}$ Der akademische Senat stimmte diesem übermittelten Vorhaben am 12. Februar $1970 \mathrm{zu}^{18}$

Der Gründung des IFSH stand zu diesem Zeitpunkt eigentlich nichts mehr im Wege. Zudem legte der Wissenschaftsrat im Mai seine Empfehlungen zur Förderung der Friedens- und Konfliktforschung vor. Aber in Hamburg ging es nicht voran. Baudissin, dem zwar von allen Seiten persönliche Integrität bescheinigt

14 Wolf Graf von Baudissin: Das Kriegsbild, in: Wehrwissenschaftliche Rundschau, 12/1962, S. 370f.; vgl. Axel Gablik: „... von da an herrscht Friedhofsruhe. " Zum Realitätsgehalt Baudissinscher Kriegsbildvorstellungen, in: Martin Kutz (Hg.): Gesellschaft, Krieg und Frieden im Denken von Wolf Graf von Baudissin, Baden-Baden 2004, S. 45ff.

15 Vgl. den Beitrag von Graf Baudissin: Bemerkungen zu den Heidelberger Thesen, in: Detlef Bald (Hg.): Europäische Friedenspolitik. Ethische Aufgaben, Baden-Baden 1990, S. 31ff., vgl. dort S. 103ff.

16 BDZ 68, 12 1/1 Wolf Graf von Baudissin: Warum ich über Strategie lese (Die Zeit, 1. Nov. 1968). Baudissin war zunächst an der Wirtschafts- und Sozialwissenschaftlichen Fakultät, dann in der Politikwissenschaft im Fachbereich Philosophie und Sozialwissenschaften.

17 BDZ 809133 Freie und Hansestadt Hamburg, Schulbehörde an Stiftung Volkswagenwerk, 16. Okt. 1969; BDZ 184002 Egon Bahr erklärte bei seiner Einführung als Institutsdirektor, auch Carl Friedrich von Weizsäcker habe sich für die Gründung des IFSH engagiert.

18 Protokolle des Akademischen Senats, vgl. Wasmuht: Geschichte, S. 255. wurde, gab den Anlass oder, besser gesagt, er bot den Anlass, gewissermaßen vor Ort die bundespolitischen Auseinandersetzungen um die Entspannungspolitik auszutragen. ${ }^{19}$ Schon mit der ersten Vorlesung war Baudissin in den zeitgenössisch mächtigen Sog inszenierter, intriganter und ideologischer Querelen von links und rechts geraten. Die universitäre Kontroverse fand eine feinsinnig mitgetragene, doch verdeckte Rückendeckung bei jüngeren, pazifistischen Abgeordneten innerhalb der SPDFraktion in der Bürgerschaft. ${ }^{20}$ Diese Fraktion selbst war zudem gespalten, den Älteren ging Baudissin mit seinem Anliegen der Rüstungsbegrenzung viel zu weit. Der „General“ wirkte bei ihnen nicht vertrauensbildend, sie misstrauten dem Friedensforscher. Den wichtigsten öffentlich und politisch wirksamen Widerstand gegen die Forschungskonzeption des Instituts trug allerdings in der CDU-Fraktion Erik Blumenfeld vor, der zum prominenten Sprecher gegen die Friedensforschung in Hamburg avancierte.

Die Proteste bewirkten lähmenden Stillstand, die vorgesehene „umgehende“ Errichtung der Stiftung des IFSH wurde auf „demnächst“ verschoben. ${ }^{21}$ Die konservative Opposition gegen die Ausweitung von Wissenschaft und Bildung an den Universitäten war in der Bonner Republik typisch für die hitzigen Kontroversen um die Lösung der deutschen „Bildungskatastrophe“, die Georg Picht erkannt hatte. ${ }^{22}$ Bildung war zum Streitpunkt der Parteipolitik auf allen Ebenen geworden, so auch in Hamburg. Das Fazit kann gezogen werden: Bildungsund Forschungspolitik führte, wie im Fall der Gründung des IFSH, zu einer hochbrisanten Kontroverse. In der Geschichte des Instituts war dies 1970 das erste Mal, es sollte nicht bei diesem einen Fall bleiben.

Das Ringen um das IFSH passt zum Gesamtbild des gesellschaftlichen Streits um Sicherheit, dass die Friedens- und Konfliktforschung unter der heftigen Gegnerschaft jener konservativen Politiker und Publizisten litt, die im wesentlichen in militärischer Stärke den wichtigsten Faktor der Friedenssicherung sahen. Sie interpretierten das Nachdenken über den Abbau von Feindbildern, über beidseitige Vertrauensbildung, Rüstungskontrolle und Abrüstung, über blockübergreifende Kooperation sowie über Konzepte einer neuen europäischen Sicherheitsordnung tendenziell als Anzeichen politischer Schwäche und bekämpften sie.

\section{Die Gründung des IFSH}

Am 11. Juni 1971 war es soweit: Die Satzung der Stiftung „Institut für Friedensforschung und Sicherheitspolitik an der Universität Hamburg " wurde erlassen und unterzeichnet. Weit wurden die Aufgaben gestellt, sich „im Rahmen der Friedensforschung

19 Vgl. Michael Staack (Hg.): Zur Aktualität des Denkens von Wolf Graf von Baudissin. Innere Führung, Hamburg 2011. Zu den Lehrveranstaltungen S. 27f.

20 BDZ 7132 Manuskript einer Sendung von Bernhard Wördehoff, NDR, 1. März 1971.

21 BDZ 71323 Behörde für Wissenschaft und Kunst an Graf Baudissin, 1. März 1971.

22 Die Wissenschaftspolitik in der Bundesrepublik hatte ihre beharrenden Seiten zu Beginn der 1950er Jahre schon gezeigt, als sich Kultusministerien und Universitäten weigerten, die Politikwissenschaft einzuführen. Erst nach dem Druck der Besatzungsmächte wurden die Lehrstühle und Institute in Berlin, Frankfurt, Freiburg und München eingerichtet. 
speziell mit sicherheitspolitischen Problemen zu befassen und dabei die Kriterien von freier Forschung und Lehre, Förderung des wissenschaftlichen Nachwuchses und Publizierung der Forschungsergebnisse zu erfüllen. "Dann wurde noch detailliert festgehalten: „Probleme der Friedenssicherung“ sowie „sicherheitspolitische Studien unter spezifisch europäischen und deutschen Aspekten“. ${ }^{23}$

Betrachtet man diesen Katalog der Satzung aus heutiger Sicht, fällt die starke Konzentration auf die sicherheitspolitische Forschung zum Frieden hin auf. Die Kriterien der Wissenschaft, Unabhängigkeit der Arbeit sowie Kritikfähigkeit der Resultate durch Offenlegen der Ergebnisse, wurden ausdrücklich aufgeführt: freie Forschung und Lehre nur in Verbindung mit dem Prinzip, die angefertigten Analysen und die angefallenen Erkenntnisse der Projekte zu publizieren. Etwas zusätzliches, über den normalen wissenschaftlichen Kanon hinausgehend, ist in dem Auftrag zu erkennen, in die Öffentlichkeit hinein zu wirken. Alle möglichen Medien werden beinah enumerativ aufgeführt. Dies kann als gesellschaftlich einmalige Verpflichtung und als eine öffentliche Verantwortung gegenüber Stadt und Land, eine besondere Verantwortung gegenüber Politik und Gesellschaft begriffen werden; sie sollte nicht vernachlässigt werden. Sie setzt der Arbeit des IFSH einen bemerkenswerten Akzent; sie ist eines ihrer Markenzeichen. Dieser Auftrag an das Institut sollte gepflegt werden.

In jenen Jahren des Institutsaufbaus, kurz bevor Dieter S. Lutz 1976 als Stellvertretender Direktor von Volker Rittberger in Tübingen nach Hamburg kam, fand ich das erste Mal den Weg ans IFSH. Ich muss gestehen, als ich nach Blankenese fuhr, hatte ich kein sicheres Bild vom Institut. Wohl wusste ich, da ich mich für die Bildungsreform der Bundeswehr engagierte, welch großer Ideengeber der Inneren Führung und des militärischen Bildungskonzept Graf Baudissin gewesen war, ${ }^{24}$ doch seine Haltung in der Sicherheitspolitik sollte ich noch kennenlernen. Immer noch kursierten bildungspolitisch gestreute Diffamierungen gegen die Friedens- und Konfliktforschung an den Universitäten, also auch gegen das IFSH; daneben die Distanz der 68er - und anderer Radikaler der Studentenbewegung - gegenüber dem Thema Militär überhaupt.

Wohin führte mich der Weg am Falkenstein: zu einem geheimen Ableger des Bundesnachrichtendienstes auf ehemaligem Militärgelände oder wirklich, wozu ich eingeladen war, zu einem wissenschaftlichen Institut der Friedensforschung? Nicht einmal die naheliegende Geschichte war bekannt, dass in der Zeit, bevor das Institut am Falkenstein einzog, der Hamburger Katastrophenschutz in diesem Haus seine Zentrale eingerichtet hatte und von dort Hamburger Geschichte geschrieben wurde; während der bedrohlichen Flutkatastrophe hatte damals Innensenator Helmut Schmidt die entsprechenden Kommunikationsmittel genutzt, als er seine Einsatzleitung hier aufgeschlagen hatte. Die historische Wirklichkeit entsprach überhaupt nicht den Unterstellungen. Das wusste ich noch nicht, als ich der Einladung von Graf Baudissin an sein Institut für Friedensforschung folgte, um über Probleme der deutschen

23 BDZ 790202 Satzung der Stiftung, § 2, Hamburg, 11. Juni 1971.

24 Vgl. Detlef Bald: Die Bundeswehr 1955-2005. Eine kritische Geschichte, München 2005. nuklearen Bündnis-Sicherheitspolitik zu sprechen. Als ich mich dem weiten Aufgang des großzügig angelegten, gediegenen Anwesens am Falkenstein näherte und die friedliche Atmosphäre wahrnahm, die jeden Besucher einladend umfing, dachte ich: Was für ein idealer Ort zu forschen! Und die Anschrift „Falkenstein“ - nomen est omen - konnte nur die Botschaft sein: mit klarem Auge die Dinge scharf in den Blick nehmen.

\section{Etappen der Institutsentwicklung}

Die Ruhe und Beschaulichkeit waren dem IFSH jedoch nicht auf Dauer vergönnt. Zweimal wurde das IFSH in seiner Geschichte durchgeschüttelt, war jeweils in seiner Konstitution betroffen, wurde um- und ausgebaut wie die gesamte Friedensforschung. Das erste Mal herrschte die breite Akzeptanzkrise der nuklearen Nachrüstung in Politik und Gesellschaft: die große Friedensbewegung der 1980er Jahre. Fachexpertise wurde gesucht - vielleicht für Lösungen oder auch zur Legitimation durch Wissenschaft. Sicherlich lag hier am Institut Kompetenz, Modelle der Rüstungssteuerung praktikabel weiter aufzubereiten und den verflixten Datensalat der Waffenarsenale zu ordnen. Doch Politik wollte mehr. Schon 1981 wurde politischer Druck derart stark ausgeübt, dass der sonst eher Freundlichkeit verbürgende Graf Baudissin diese Ansprüche, in die Forschung einzugreifen, gegenüber dem Bürgermeister schroff zurückwies. Er werde „ein technokratisches Institut für Abrüstungsfragen“ ähnlich dem in Ebenhausen, das für Politik und Kanzleramt vertrauliche Gutachten liefere, nicht mittragen. ${ }^{25}$

Nach einem Jahr Verhandlungen gelang dann ein Konsens: Einerseits würde das Institut die Aufgaben hinsichtlich „Abrüstungspolitik in Europa fortentwickeln“ ${ }^{26}$ und andererseits gab Hamburg die Zusage, die Zahl der Mitarbeiter etwa zu verdoppeln und den Institutshaushalt um die Hälfte zu erhöhen. Damit hatte die alte Klage Baudissins Erfolg, die personelle Ausstattung des IFSH sei „hängengeblieben“27. Das entsprach den Sachverhalten und den Empfehlungen zur inhaltlichen Ausrichtung der Schwerpunkte, die der Wissenschaftsrat im Mai 1983 vorlegte: „Diese insgesamt begrenzten personellen und finanziellen Ressourcen sind nicht hinreichend, die bestehenden Forschungslücken zu existentiellen Fragen der Friedenspolitik zu füllen [und, DB] umsetzungsfähige Vorschläge zu erarbeiten...“. IFSH und Stadt begrüßten diese Entwicklung, das Thema „kooperative Rüstungssteuerung“ in neuen Projekten „unter Berücksichtigung spezifisch europäischer und deutscher Aspekte" anzugehen. ${ }^{28}$

Die zweite große Unruhe- und Prüfungsphase des IFSH liegt keine zehn Jahre zurück. Auf den Bologna-Prozess und die PisaBildungskatastrophe reagierte das Institut zeitgemäß; Dieter S. Lutz mobilisierte die Kräfte. Unter seiner Leitung wurden die universitäre Lehre und die Förderung des wissenschaftlichen Nachwuchses strukturell intensiviert. Das Doktorandensemi-

25 BDZ 790203 Wolf Graf Baudissin an Klaus von Dohnanyi, 18. Febr. 1982. 26 Regierungserklärung des 1. Bürgermeisters, 23. Febr. 1983.

27 Wolf Graf Baudissin: Probleme der Friedensforschung, in: Peter Lock (Hg.): Frieden als Gegenstand der Wissenschaft, Frankfurt/M. 1982, S. 9.

28 BDZ 184006 Senatsdrucksache (über den Ausbau des IFSH, Sept.? 1983). Im Haushaltsansatz für das Institut wurden fünf weitere Stellen für Wissenschaftler vorgesehen. 
nar fand mehr und neue Aufmerksamkeit, aber mit Priorität wurde der Masterstudiengang (M.P.S. - Peace and Security Studies) entworfen und bereits im Wintersemester 2002 gestartet. Alles schien gut gefügt und in Gang gesetzt, wenn nicht die Politik wieder ihr Spiel mit der Bildungspolitik getrieben hätte. Eine veränderte politische Konstellation im Rathaus erzeugte Irritationen über die Zukunft des Instituts, was, von außerhalb Hamburgs betrachtet, kaum sachlich begründet, sondern wie ein Rückfall in jene Verhältnisse zu sein schien, als sich drei Jahrzehnte zuvor „rechte“ Politik gegen kritische Wissenschaft stellte. Gleichwohl, die fachliche Leistung des Instituts fand Anerkennung. Dank der nicht versiegenden Überzeugungskraft von Reinhard Mutz, der die Institutsbelange verantwortete, gelang es, dem IFSH die notwendige Luft zu erhalten, um zu arbeiten, zu forschen und zu lehren. Es gab dann erneut die Stabilisierung, es folgte eine programmatische Erneuerung und institutionelle Stärkung, als im Frühjahr 2007 das Forschungszentrum „Beim Schlump“ im Bereich der Universität bezogen wurde.

\section{Freie und angewandte Friedensforschung}

Das Institut hat von Anbeginn an die Bedingungen der Wissenschaft erfüllt. Innerhalb weniger Jahre konnte es zu einem Dialogpartner von internationalem Rang wachsen, auf der Bühne akademischer Kritik und Konkurrenz war es exzellent etabliert, die Leistung hoch, die Erwartungen mehr als erfüllt. Diese Würdigung gilt auch, wenn wir einen Blick werfen auf das andere große deutsche Institut in Frankfurt, wo etwa zeitgleich, am 30. Oktober 1970, die „Hessische Stiftung Friedens- und Konfliktforschung" das Licht der akademischen Welt erblickte. Es hatte, recht betrachtet, einen vergleichsweise leichteren, günstigeren Beginn. Ihr besonderes Startkapital zeichnete sich aus durch einige, in der internationalen Politik bewanderter und bereits ausgewiesener, universitär integrierter Wissenschaftler wie Ernst-Otto Czempiel, Klaus-Jürgen Gantzel, Hans Nicklas und Dieter Senghaas. Auch wenn die HSFK ihrer Arbeit einen breiteren Friedensbegriff zugrunde legte, gehörte zu ihrem Aufgabenspektrum auch eine klare Praxisorientierung. Sie sollte innovativ „Transformations- und Lösungskonzepte entwickeln“. ${ }^{29}$ In der Rüstungsdynamik des Ost-West-Konflikts suchte man in Frankfurt mit Lösungsvorschlägen Politik „beeinflussen“ zu können. ${ }^{30}$ Ähnlich untersuchte man in Hamburg die „kooperative Transformation des europäischen Systems internationaler Beziehungen“, wie die Hamburger Bürgerschaft zufrieden feststellen konnte. ${ }^{31}$

Politikberatung stand bei beiden Instituten auf der Agenda. Von Anbeginn an gehörte Praxisorientierung zur Friedens- und Konfliktforschung - um einer politischen Kultur des Friedens zu dienen. Beim Besuch beider Institute konnte man in den Jahren leicht hören, wie subtil man hier metatheoretische und methodische Diskurse führe oder im Unterschied dazu dort inhaltliche Phasen und thematische Setzungen erkenne, um

29 Verfassung der HSFK, zitiert nach Wasmuht: Geschichte, S. 243.

30 Gert Krell: Friedensforschung in Hessen. Zur Geschichte und Entwicklung der HSFK, Frankfurt/M. 1987, S. 16

31 BDZ 150006 Bürgerschaft der Freien und Hansestadt Hamburg, Protokolle, 8/2118, S. 3 (Fünf Jahre IFSH). die spezifische Andersartigkeit von IFSH und HSFK zu zeichnen. Diese Wertung der eigenen Standortbestimmung sollte man sorgsam beachten, aber nicht überschätzen. Denn manche Entwicklung erklärte sich ganz einfach, da oder wenn aus pragmatischen oder persönlichen Gründen der einzelnen Wissenschaftler ein Thema einfach aus der realen zeithistorischen Politik wählte.

Praxisbezug also konstituierte von Anfang an die Friedens- und Konfliktforschung. „Alles hat seine Zeit“, wie Dieter Senghaas die Phasen und die Aktivitäten der Friedensforschung einordnete, auch die Beratung politischer Entscheidungsträger und die politische Praxisorientierung. ${ }^{32}$ Graf Baudissin war dafür offen, er hatte Erfahrung darin gesammelt. So war es neben Aufklärung auch sein Anliegen, wenngleich auch Skepsis hinsichtlich der Wirkung und Reichweite bezüglich der Politikberatung aufschimmert. Realistisch wie er war, befürchtete er eher „einen langen, mühseligen Transformationsprozess voller Rückschläge“. Doch er sah ihre Chancen, wenn sie „zu vertieftem Verständnis von Ursachen, Abläufen und Regelungen von Konflikten führt und bestehende Konfliktlösungsmuster zur Diskussion stellt, liefert sie Beurteilungsmaßstäbe und Entscheidungshilfen, die für eine problembewusste politische und militärische Führung von großer Bedeutung sein können. " 33

Aus diesen Formulierungen ist wenig Zuversicht zu spüren. Allein im Konjunktiv schimmert ein wenig Hoffnung mit, während Graf Baudissin doch zur gleichen Zeit Kanzler Schmidt beratend zur Seite stand, in Helsinki den Korb der „Vertrauensbildenden Maßnahmen“ für die Schlussakte der Konferenz über Sicherheit und Zusammenarbeit 1974 zu schnüren. In Maßen legte Graf Baudissin allerdings darauf großen Wert, da er überzeugt war, die internationale beidseitige Kooperation der Experten der Militärelite würde die Mauern des Antagonismus in den Köpfen abbauen und die alten Denkschemata unterminieren. ${ }^{34}$ Das würde auch in der Bundesrepublik wirken und der Bundeswehr helfen. Der Erfolg konnte langsam keimen und beitragen, das „Instrument kooperativer Rüstungssteuerung“ in der Diplomatie und den Militärbürokratien voranzubringen. ${ }^{35}$

Als dann Egon Bahr 1984 die Verantwortung im Institut übernahm, lagen die Schatten der heißen Phase der nuklearen Aufrüstung über Europa. Kennzeichen dieser Jahre waren Proteste gegen Atomrüstung mit großen gesellschaftliche Turbulenzen und beeindruckenden Demonstrationen der Friedensbewegung ebenso wie die nicht endenden Expertisen der Friedensforschung über nukleare Szenarios und Siegstrategien durch Atomwaffeneskalation - nebenbei ein Thema, das nicht aus der Welt ist. Es berührt auch Deutschland immer noch direkt; auch 2011 besteht die Bundesregierung auf der Teilhabe von Atomwaffen für die Bundeswehr und für die NATO-Strategie.

32 Dieter Senghaas: Der Frieden und seine Erforschung. Bilanz eines halben Jahrhunderts Friedensforschung, in: Blätter für deutsche und internationale Politik, Jg. 35, 12/2010, S. 85

33 BDZ 157001 Wolf Graf Baudissin an N., 12. Aug. 1974

34 Vgl. Detlef Bald: Hiroshima 6. August 1945. Die nukleare Bedrohung, München 1999, S. 152.

35 Wolf Graf Baudissin: Vertrauensbildende Maßnahmen als Instrument kooperativer Rüstungssteuerung, in: Jost Delbrück, Norbert Ropers, Gerda Zellentin (Hg.): Grünbuch zu den Folgewirkungen der KSZE, Köln 1977. 
Dieser Thematik wird in der Friedensforschung gegenwärtig viel zu wenig Beachtung geschenkt.

Doch in den 1980er Jahren waren sektorale Aufrüstung, nukleare Selbstabschreckung, strukturelle Nichtangriffsfähigkeit Stichworte eines „Euroshima“, der nuklearen Vernichtung des Kontinents Europa wie in Hiroshima. Konventionelle Abrüstung und Denuklearisierung schimmerten am politischen Horizont der Hoffnung. Die Antwort auf diese Unübersichtlichkeit lag in der Besinnung auf Frieden und Stabilität. Die Erfahrungen mit „Wandel durch Annäherung“ inspirierten das Modell internationaler Kooperation für „Gemeinsame Sicherheit“ in einer neuen europäischen Friedensordnung. Das nahm Egon Bahr zum Auftrag, sein Jahrzehnt der Institutsleitung diesem Projekt eines europäischen Friedens vom Atlantik bis zum Ural zu widmen und dafür in der Politik zu werben. Die scheinbar so festgefügte Abschreckungsarchitektur des Kalten Krieges zeigte Risse, bevor die Säulen der Bedrohung endlich im Jahr 1990 zusammenbrachen. Egon Bahr fand in der europaweiten Demilitarisierung, um eine übergreifende Friedensordnung in ganz Europa anzulegen, einen Mitstreiter in Dieter S. Lutz. Er, der jahrelange Stellvertreter, übernahm 1994 die volle Verantwortung des IFSH. Festzuhalten ist, der Forschungsschwerpunkt der Transformation durch Kooperation in Europa begleitete das IFSH seit den ersten Tagen der 40-jährigen Geschichte bis heute; es ist ein Thema - weit über die OSZE hinausreichend - und wird eine tragende Säule des Forschungsprofils bleiben.

\section{Bundeswehr im Forschungskonzept}

Eine andere Säule der Aufgaben des IFSH lag in der Beachtung des spezifischen „deutschen Aspekts“ der Sicherheitspolitik, der Bundeswehr. Unter allen Friedensforschungsinstituten ist dies einmalig. Die Bundeswehr ist kein fernes Phänomen der Betrachtung, sondern konkreter Gegenstand der Begleitung, vor allem aber der wissenschaftlichen Analyse. Der Faktor Militär als Subjekt und Objekt der Sicherheitspolitik unterstreicht, dass Friedensforschung zur Sicherheitspolitik nicht auf Regierungshandeln oder Rüstungsdaten zu beschränken ist.

Graf Baudissin hat dieses Thema aus verständlichen Gründen nicht in den Vordergrund gestellt, zu sehr war „Bundeswehr“ mit seinem Namen verbunden. Daher konzentrierte er sich auf die übergeordnete bündnisbestimmte Strategie. Aber er hatte bei der internationalen Kooperation im Helsinki-Prozess doch den kleinen Nebenaspekt im Blick, in Deutschland ein „friedensbewusstes kritisches, mit Zivilcourage ausgestattetes Offizierkorps heranzubilden und auf diese Weise den im Atomzeitalter noch wichtigeren Aufgaben der Kriegsverhütung eine Grundlage im politischen System zu verschaffen. “36 Als ehemaligem Soldat war Graf Baudissin die Problemebene, die allgemeine politische und internationale Kompetenz der Bundeswehroffiziere auszuweiten und ein höheres Niveau anzustreben, nur zu bewusst.

36 Jürgen Reusch: Friedensforschung in der Bundesrepublik. Entwicklung, Positionen, Perspektiven, Frankfurt/M. 1986, S. 88; die ersten eingestellten Mitarbeiter des IFSH waren Lothar Wilker, danach Rüdiger Jütte, Arno Burzig, Annemarie Große.
Aus langen Einblicken in die Arbeit des Instituts kann ich nur anerkennen, es ist eine eigene Art der Beziehung zur Bundeswehr entwickelt worden. Die gefundene Symbiose erscheint mir fruchtbar, natürlich auch nicht spannungsfrei, aber im Interesse beider Seiten fortzuführen. Aus der Forschungsperspektive eröffnet sich ein weites Betätigungsfeld. Gerade nach den Umbrüchen 1990/94 mit der Bildung der „Armee der Einheit“, dem Wegfall des Typs der Massenarmee zugunsten einer global interventionsfähigen „Armee im Einsatz“ ist sogar ein weites Feld an Problemen von Staat, Macht und Frieden gegeben, um einen eigenen Forschungsschwerpunkt zu konzipieren. Die Politik hat programmatisch Konturen der Macht aufgebaut, die herausfordern.

Die deutsche „Verantwortung in der Welt“ wurde von Bundeskanzler Helmut Kohl am Tag nach der Einheit am 4. Oktober 1991 proklamiert. Zwei Jahrzehnte später, im Mai 2011 hat Verteidigungsminister Thomas de Maizière die Konsequenzen in der Sicherheitspolitik dahin gezogen, es gehe um „Deutschlands Platz in der Welt“ und um die „Interessen als starke Nation in der Mitte Europas“. Was für ein nationalstaatlicher Kontext? Soll damit die postnationale Logik machtpolitischer Interessendefinition aufgegeben werden, die vor einem Jahrzehnt den globalen Interventionismus begründete, um mit militärischen Mitteln Menschenrechte und Humanismus global zu vertreten. ${ }^{37}$ De Maizière stellte mit diesen Formulierungen fest: Deutsche „Streitkräfte sind unentbehrliches Instrument der Außen- und Sicherheitspolitik unseres Landes“. Dieser Stellenwert ist neu, zuvor so nicht vertreten. Ebenso überraschend gewann Bündnissolidarität den Wert einer „Staatsräson“ - so präzisierte er offiziell die Aufgaben der „Armee im Einsatz“. ${ }^{38}$

Staatsräson, was heißt das? Deutsche Interessen müssen Gegenstand der Forschung sein, welche Interessen sind gemeint und wie stärken sie nachhaltig den Frieden in Europa? Welche Ethik kann oder soll Außenpolitik leiten - was will und bedeutet deutsche Staatsräson, gegenüber der NATO und nicht für Europa? Der Friedensauftrag des Grundgesetzes kann auch hier Anstoß sein und Maß setzen. Friedensforschung ist herausgefordert, die Waage zwischen formaler Legalität und humaner Legitimität auszutarieren. Der Diskurs über Macht und ethische Forderungen, wie schon das Bundesverfassungsgericht sowie die Bundespräsidenten Roman Herzog und Horst Köhler anmahnten, ist der Friedensforschung aufgegeben. Die Klagen über die unübersichtliche Sicherheitspolitik sind Legion. Friedensforschung sollte das heiße Eisen anpacken und Grundlagenarbeit leisten.

\section{Und morgen?}

Welche Idylle, welche Abgeschiedenheit strömte das Haus am Falkenstein aus. Doch dies ist abgeschlossen, ist Geschichte. Nun - Beim Schlump - wohin werden die kommenden Jahre

37 Vgl. die Diskussion zwischen Ulrich Beck: Über den postnationalen Krieg, in: Blätter für deutsche und internationale Politik, Jg. 24, 8/1999, S. 984ff. und Jürgen Habermas: Bestialität und Humanität, in: Die Zeit, 29. April 1999; die Weiterentwicklung: Ulrich Beck: Empört Euch, Europäer. Zusammen gewinnen oder einzeln verlieren, in: Der Spiegel, 34/2011, S. 128ff. und Jürgen Habermas: Zur Verfassung Europas, Berlin 2011.

38 Eckpunkte für die Neuausrichtung der Bundeswehr, Berlin, 18. Mai 2011. 
führen? Das Getriebe der Universität und des modernen Wissenschaftssystems birgt vielerlei Anreize, manche entdecken die Fallen des Systems, auch die Belastungen. Die Herausforderungen sind zu meistern. Die universitäre Welt des IFSH ist voller Chancen. Es geht um die Zukunft: Michael Brzoska hat als Direktor ausreichend zu tun: Integration und Kooperation, Besprechungen und Veranstaltungen, Rationalisierung und Budgetierung, Vernetzung und Lehrauftrag - nicht nur leere Begriffe. Solide und angemessene Grundlagen der Finanzierung sind vonnöten und mit Phantasie strategisch zu erschließen - schwierig genug. Es ist viel anzupacken, die personellen, logistischen und verwaltungsmäßigen Bedingungen dieses großen Instituts zu bewältigen und zu lösen.

Und dann steht auch die Zukunft der Friedensforschung am IFSH in der Debatte: Welches Erkenntnisinteresse leitet die theoretische Reflexion der Analyse der Einzelphänomene oder was soll kritische Friedensforschung wissenschaftlich leisten? Welcher Friedensbegriff leitet die Forschung, welcher kann für praktische Politik sinnstiftend sein? Damit sich auseinanderzusetzen ist kein Glasperlenspiel im akademischen Elfenbeinturm das sind existenzielle Themen der Zukunft. Friedensforschung ist „Zukunftsforschung“, wie bei der Gründung gesagt; Frieden und Sicherheit bleiben unter „europäischen und deutschen Aspekten“ im Fokus. War es im Kalten Krieg der Königsweg, das nukleare Bedrohungs- und Feindbildszenario durchsichtig und politisch handhabbar zu machen, so steht nun die Frage im Raum: Was ist heute der Königsweg hin zu einer lebenswürdigen Friedensordnung in Europa und hin zu einer Kultur des Friedens? Was bleibt - herausfordernd - die Vision eines gerechten Friedens?

Wie am Anfang am Falkenstein: die Tradition aufgreifend, der scharfe Blick des Falken für eine treffliche, sinngebende Friedens- und Zukunftsforschung.

\section{Internationale}

\section{Entwicklungszusammenarbeit}

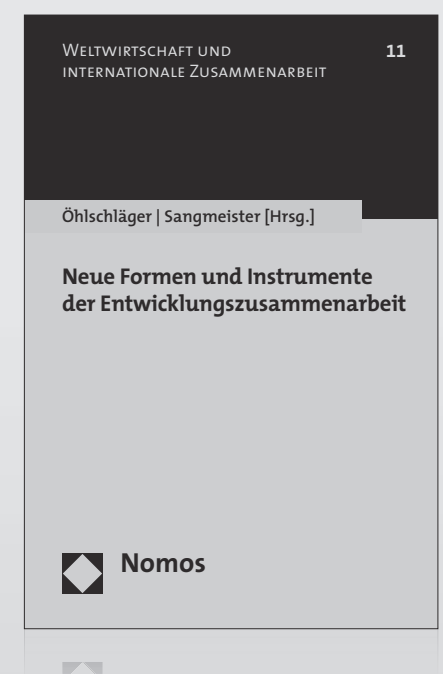

\footnotetext{
Neue Formen und Instrumente der Entwicklungszusammenarbeit

Herausgegeben von Rainer Öhlschläger und Hartmut Sangmeister

2012, 188 S., brosch., 32,-€

ISBN 978-3-8329-6948-6
}

(Weltwirtschaft und internationale

Zusammenarbeit, $B d$. 11)

Der Sammelband analysiert neue Strategien, Formen und Instrumente der internationalen Entwicklungszusammenarbeit, für die Armutsbekämpfung nach wie vor an erster Stelle steht. Die internationale Zusammenarbeit sieht sich aber auch mit neuen Herausforderungen wie Terrorismus, Migrationsströme, Konflikte um Wasser und Klimawandel konfrontiert.

Weitere Informationen: www.nomos-shop.de/14048

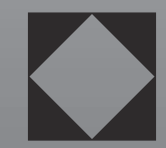

